\title{
MUTYH Mutations Do Not Cause HNPCC or Late Onset Familial Colorectal Cancer
}

\author{
Astrid Stormorken', Karen-Marie Heintz², Per Arne Andresen', Eivind Hovig'², Pål Møller' \\ ISection for Inherited Cancer, Department for Medical Genetics, Rikshospitalet-Radiumhospitalet Medical Center, Oslo, Norway; ${ }^{2}$ Department of Tumour Biology, Institute for Cancer \\ Research, Rikshospitalet-Radiumhospitalet Medical Center, Oslo, Norway; ${ }^{3}$ Department of Pathology, Rikshospitalet-Radiumhospitalet Medical Center, Oslo, Norway
}

Key words: familial adenomatous polyposis coli, hereditary non-polyposis colorectal cancer, late onset familial colorectal cancer, MUTYH mutations

Corresponding author: Pål Møller, Section of Genetic Counselling, Department of Cancer Genetics, The Norwegian Radium Hospital, N-0310 0slo, Norway, tel.: +47229356 75, fax: +47229352 19,e-mail:pal.moller@medisin.uio.no

Submitted: 7 April 2006

Accepted: 20 May 2006

\begin{abstract}
Recently, carriers of biallelic mutations in the base excision repair gene MUTYH, have been demonstrated to have a predisposition for multiple adenomas and colorectal cancer. Still, many questions remain unanswered concerning MUTYH. We have addressed the following: Do biallelic MUTYH mutation carriers invariably demonstrate FAP, and may MUTYH be a gene causing HNPCC, HNPCC-like or dominantly inherited late onset colorectal cancer? We examined affecteds from our total series of HNPCC, HNPCC-like and dominantly inherited late onset colorectal cancer kindreds not demonstrated to have any MMR mutations. Bloodsamples from 96 patients were subjected to sequencing of exon 7 and exon 13 in the MUTYH gene. Two heterozygotes and one homozygote for the European founder mutations were found. The homozygous carrier did not meet criteria for FAP/AFAP. We conclude that MUTYH, when mutated, causes a rare recessively inherited disorder including colorectal- and duodenal cancers. It is not verified that heterozygous carriers of MUTYH mutations have an increased risk of cancer, and they do not explain the occurrence of familial colorectal cancer in the population.
\end{abstract}

\section{Introduction}

Recently, carriers of biallelic mutations in the base excision repair gene, MUTYH at 1p34.1, have been demonstrated to have a predisposition for multiple adenomas and colorectal cancer (CRC) causing autosomal recessive inherited disease [1]. The gene encodes a glycolase which is involved in repair of DNA damage caused by reactive oxygen species generated during aerobic metabolism [2, 3]. In variant forms this ability is reduced [4]. Oxidative damage to DNA can lead to degenerative diseases, aging and cancer [2].

Sequence variants in MUTYH are found in different ethnic groups [1]. Two common MUTYH mutations,
Y165C and G382D, account for approximately 85\% of the mutations occurring in individuals of Caucasian ancestry [1]. Other MUTYH mutations known to be associated with colorectal cancer (CRC) are E466X, Y90X and 1395delGGA [1, 5]. Combined data from previous studies suggest that about $1 \%$ of colorectal cancer cases can be attributed to biallelic MUTYH mutations [3, 6-8]. Families classified as Familial Adenomatous Polyposis (FAP) or Attenuated FAP (AFAP), but without a detected mutation in the Adenomatous Polyposis Coli (APC) gene, may be caused by biallelic MUTYH mutations [9].

Phenotypic expression of the MUTYH mutation in the heterozygous form is not clear. Kairupan et al. [9] 
demonstrated that the MUTYH mutations Y165C, G382D and 1395delGGA were not present in the APC mutation-positive FAP population they investigated, suggesting that it does not act as a modifier gene in the development of FAP. The mismatch repair genes also have roles in the removal of oxidative damage [10]. It is demonstrated that the MSH2/MSH6 complex is physically associated with MUTYH at an MSH6 binding site and is required to stimulate MUTYH activity $[10,11]$. Disruption of the MSH2/MSH6 complex causes HNPCC, and it has been reported that MUTYH mutations are associated with colorectal cancer risk and can be associated with the clinical features of HNPCC [9, 12]. Kambara et al. [8] suggest that MUTYH variation does not initiate progression of cancer, but acts as a promoter in a subset of CRCs. Croitoru et al. [13] demonstrated that heterozygous mutations in $\mathrm{Y} 165 \mathrm{C}$ and $\mathrm{G} 382 \mathrm{D}$ may confer a potentially important low-penetrant risk of CRC.

A number of families fit the clinical criteria for FAP, AFAP, HNPCC or appear to be inherited, but mutations in known genes have not yet been found. About 30\% of FAP cases are apparently "sporadic" (new mutations) giving a one in two risk to their offspring, but low risk to their siblings. If these cases are caused by MUTYH mutations and recessive inheritance, the risk to siblings is one in four and the risk to offspring is low in small sibships. The findings have clinical implications for the families as to evaluating the risk of developing cancer and mode of surveillance.

Many questions remain unanswered concerning MUTYH. We have addressed the following: 1) Do biallelic MUTYH mutation carriers invariably demonstrate FAP? 2) May MUTYH be a gene causing HNPCC, HNPCC-like or dominantly inherited late onset colorectal cancer (LOCRC)?

Previously $[14,15]$ we have identified a number of families with aggregation of CRCs and/or colorectal polyps. We have used the tools available to identify known CRC syndromes in these families, but in a majority no definite cause has been demonstrated. We here report the results of screening these families for MUTYH mutations.

\section{Patients and methods}

The patients included in the study had been referred to the Section of Genetic Counselling at the Norwegian Radium Hospital. They were included and classified according to their family history of colorectal and other cancers applying wide clinical criteria [16]. The family history was expanded and information on the site and classification of cancers and polyps and age at diagnosis were obtained and verified in the medical files and/or in the National Cancer Registry whenever possible.

We examined affecteds from our total series of HNPCC, HNPCC-like and LOCRC kindreds demonstrated not to have any MMR mutations. Blood samples from 96 patients diagnosed with either cancer and/or tubular adenomas were examined. Thirty-one belonged to HNPCC families, i.e. they met the Amsterdam criteria II [17]; 43 were HNPCC-like, i.e. they almost met the Amsterdam criteria II and/or had cases with multiple primaries; and 22 belonged to families with LOCRC, i.e. presence in the family of four or more HNPCCrelated cancers all diagnosed over 50 years of age [18]. Sixty-three index persons had at least one cancer and 33 index persons had one or more adenomas. Median age of first tumour was 49 years (23-78) for colorectal cancers $(n=53), 49$ years (29-76) for tubular adenomas $(n=33), 54$ years (44-62) for endometrial cancers $(n=4)$ and 57 years (39-69) for other cancers $(n=6)$.

Tumours from eighty-five affecteds had been subjected to immunohistochemistry (IHC) for the presence of $\mathrm{MLH} 1, \mathrm{MSH} 2$ and MSH6 gene products using standard procedures [14, 15, 19, 20]. Blood samples from all patients demonstrating lack of gene product in tumour by IHC $(n=66)$ had been subjected to sequencing of the corresponding gene and searching for large rearrangements in $M L H T$ and MSH2 [14, 15, 21$]$.

All blood samples were subjected to sequencing of exon 7 (Y165C) and exon 13 (G382D) in the MUTYH gene with PCR reactions as previously described [6]. DNA sequencing was performed using a DYEnamic ET dye terminator cycle sequencing kit on a MegaBACE ${ }^{\mathrm{TM}} 1000$ (Amersham Biosciences, Uppsala, Sweden).

The medical files verifying diagnoses were obtained with written permission from living persons, or with permission from descendants of the dead ones. Written informed consent was underlying all requests for tumour specimens. If the patient was dead, the living relative(s) at risk consented. For mutation analyses, informed consent was obtained in writing following genetic counselling. Because all activity reported was approved health care, all information was kept in our medical files.

\section{Results}

Two heterozygotes for the European founder mutations were found:

- one patient was heterozygous for G382D. She had an ovarian cancer at the age of 69 and one colon adenoma. Her daughter had three tubular adenomas 48 years old, three tubular adenomas 49 years old 
and one at the age of 56, but a blood sample was not available. The family had been classified as HNPCC-like. Immunohistochemical staining with antibodies against $\mathrm{MLH} 1, \mathrm{MSH} 2$ and $\mathrm{MSH} 6$ proteins of the patient's ovarian cancer showed presence of all three proteins and mutation analyses for MMR genes were not undertaken;

- the other patient was heterozygous for $\mathrm{Y} 165 \mathrm{C}$. He had two tubular adenomas at the age of 49 and a hypophyseal adenoma at 50 . His two siblings had not had cancers or adenomas. His mother had CRC at 40 years, and endometrial cancer at 50. She developed duodenal and jejunal polyps demonstrated to include two invasive cancers, and was demonstrated to be homozygous for Y165C. Her sister had contracted CRC at 42. The family had been classified as close to the Amsterdam criteria II [17].

\section{Discussion}

In our series, selected to have dominantly inherited colorectal cancer, or suspected to have, we found no excess of MUTYH mutations. We concluded that the clustering of colorectal cancers in the families was not caused by MUTYH mutations.

The prevalence of MUTYH mutations in the series examined was $2 / 96=2 \%$, which is not more than expected by chance alone. Both mutation carriers were selected because of presence of adenomas, which consequently could not be included in the analysis of the mutation carriers. The major clinical findings were, however, that one of the patients had ovarian cancer and the other a hypophyseal adenoma. In addition, the latter had a homozygous mother with HNPCC-like phenotype including endometrial carcinoma, who later in life presented with AFAP-like disease. None of the findings indicated that MUTYH might cause dominantly inherited HNPCC or LOCRC, and none of the families included relatives with FAP.

Other studies have shown 1.35-1.67\% Y165C and G382D mutations in a control population $[9,12,13]$, $1.6 \%$ in HNPCC patients [12] and $2-2.34 \%$ in CRC patients [7, 13]. The numbers are probably underestimated since the mutation screening methods do not detect all disease-causing sequence changes. The carrier frequency in British and North American populations has been estimated to be $2 \%[4,22]$.

In the first reports on homozygous for MUTYH mutations having colorectal cancer, patients were selected on the basis of having multiple polyps (AFAP). The two heterozygous mutation carriers and also the homozygous mutation carrier in our study only had a few adenomas. Others have also shown that heterozygous and homozygous mutation carriers are not consistent with multiple adenomatous polyps $[6,7,12$, $13,23]$. It seems like homozygous for MUTYH mutations may present AFAP, but they do not invariably do so.

The homozygous (Y165C/Y165C) mutation carrier did not meet the diagnostic criteria for FAP, but developed duodenal and jejunal polyps. This is keeping with other reports: [12, 13]. Nielsen et al. [24] revealed several cases of gastro-duodenal polyps in homozygous mutation carriers in MUTYH-associated polyposis coli and Kairupan et al. [9] reported one patient with a duodenal polyp.

Farrington et al. [23] demonstrated that heterozygous mutations predispose to CRC later in life, but the effect was small and of borderline significance. Croitorv et al. [13] reported that heterozygous MUTYH mutations (Y165C and G382D) may confer a potentially important low-penetrance risk of CRC. Such reports should be interpreted with caution, because when an MUTYH mutation is found in a colorectal cancer kindred, half of the affected relatives are expected to have the same mutation. One might have expected more reports if the positive finding were true. It is possible that negative findings have not been reported. None of our LOCRC families demonstrated MUTYH mutations.

In conclusion, our findings were in keeping with our interpretation of the literature: homozygous carriers of MUTYH mutations are at increased risk of colorectal cancer. They may present FAP or AFAP, but they do not invariably do so. Heterozygous carriers of MUTYH mutations may or may not have slightly increased risk of colorectal cancer, but they do not explain the occurrence of familial colorectal cancer in the population. From a practical clinical genetic point of view, MUTYH mutations cause a rare autosomally recessively inherited disorder characterized by colorectal and duodenal cancers.

\section{Acknowledgements}

We are indebted to: all participating family members. Eldbiørg Hanslien and Toril S. Gytri, the Norwegian Radium Hospital (technical assistance).

\section{References}

1. Jones S, Emmerson P, Maynard J, Best JM, Jordan S, Williams GT, Sampson JR and Cheadle JP. Biallelic germline mutations in $\mathrm{MYH}$ predispose to multiple colorectal adenoma and somatic G:C $\rightarrow$ T:A mutations. Hum Mol Genet 2002; 11: 2961 -2967.

2. Ames BN and Gold LS. Endogenous mutagens and the causes of aging and cancer. Mutat Res 1991; 250: 3-16.

3. Halford SER, Rowan AJ, Lipton L, Sieber OM, Pack K, Thomas HJW, Hodgson SV, Bodmer WF and Tomlinson PM. Germline mutations but not somatic changes at the MYH locus contribute to the pathogenesis of unselected colorectal cancers. Am J Pathol 2003; 162: 1545-1548. 
4. Al-Tassan N, Chmiel NH, Maynard J, Fleming N, Livingston AL, Williams GT, Hodes AK, Rhodri Davies D, David SS, Sampson JR and Cheadle JP. Inherited variants of MYH associated with somatic $\mathrm{G}: \mathrm{C} \rightarrow \mathrm{T}: \mathrm{A}$ mutations in colorectal tumours. Nat Genet 2002; 30: 227-232

5. Gismondi V, Meta M, Bonelli L, Radice P, Sala P, Bertario L, Viel A, Fornasarig M, Arrigoni A, Gentile M, Ponz de Leon M, Anselmi L, Mareni C, Bruzzi P and Varesco L. Prevalence of the Y165C, G382D and 1395delGGA germline mutations of the $\mathrm{MYH}$ gene in Italian patients with adenomatous polyposis coli and colorectal adenomas. Int J Cancer 2004; 109: 680-684.

6. Enholm S, Hienonen T, Suomalainen A, Lipton L, Tomlinson I, Käriä V, Eskelinen M, Mecklin J-P, Karhu A, Järvinen HJ and Aaltonen LA. Proportion and phenotype of MYH-associated colorectal neoplasia in a population-based series of Finnish colorectal cancer patients. Am J Pathol 2003; 163: 827-832.

7. Fleischmann C, Peto J, Cheadle J, Shah B, Sampson J and Houlston RS. Comprehensive analysis of the contribution of germline MYH variation to early-onset colorectal cancer. Int J Cancer 2004; 109: 554-558.

8. Kambara T, Whitehall VL, Spring KJ, Barker MA, Arnold S, Wynter CV, Matsubara N, Tanaka N, Young JP, Leggett BA and Jass JR. Role of inherited defects of MYH in the development of sporadic colorectal cancer. Genes Chromosomes Cancer 2004; 40: 1 -9.

9. Kairupan CF, Meldrum CJ, Crooks R, Milward EA, Spigelman AD, Burgess B, Groombridge C, Kirk J, Tucker K, Ward R, Williams R and Scott RJ. Mutation analysis of the MYH gene in an Australian series of colorectal polyposis patients with or without germline APC mutations. Int J Cancer 2005; 1 16: 73-77.

10. Gu Y, Parker A, Wilson TM, Bai H, Chang D and Lu A. Human MutY Homolog, a DNA glycosylase involved in base excision repair, physically and functionally interacts with mismatch repair proteins human MutS homolog 2/Human MutS homolog 6. J Biol Chem 2002; 277: $11135-11142$.

11. Bai H, Jones S, Guan X, Wilson TM, Sampson JR, Cheadle JP and Lu A. Functional characterization of two human MutY homolog (hMYH) missense mutations (R227W and V232F) that lie within the putative hMSH6 binding domain and are associated with hMYH polyposis. Nucleic Acids Res 2005; 33: 597-604.

12. Ashton KA, Meldrum CF, McPhillips ML, Kairupan CF and Scott RJ. Frequency of the common MYH mutations (G382D and Y165C) in MMR mutation positive and negative HNPCC patients. Hereditary cancer in clinical practice 2005; 3: 65-70.

13. Croitoru ME, Cleary SP, di Nicola N, Manno M, Selander T, Aronson M, Redston M, Cotterchio M, Knight J, Gryfe R and Gallinger S. Association between biallelic and monoallelic germline MYH mutations and colorectal cancer risk. J Natl Cancer Inst 2004; 96: 1631-1634.

14. Stormorken AT, Müller W, Lemkemeyer B, Apold J, Wiinen J, Fodde R, Möslein $G$ and Møller P. Prediction of the outcome of genetic testing in HNPCC kindreds using the revised Amsterdam criteria and immunohistochemistry. Fam Cancer 2001; 1: 169-173.

15. Stormorken A, Bowitz-Lothe IM, Norén T, Kure E, Aase S, Wijnen $\mathrm{J}$, Apold J Heimdal K and Møller P. Immunohistochemistry identifies carriers of mismatch repair gene defects causing HNPCC. J Clin Oncol 2005; 23: 4705-12.

16. Møller P. Genetic cancer: a challenge and a possible strategy. J Cancer Care 1993; 2: 94-99.

17. Vasen FA, Watson P, Mecklin J-P, Lynch HT and the ICG-HNPCC. New clinical criteria for hereditary non-polyposis colorectal caner (HNPCC, Lynch syndrome) proposed by the international collaborative group on HNPCC. Gastroenterol 1999; 116: 1453-1456.
18. Norwegian Gastrointestinal Cancer Group (NGICG). Norwegian. http://ngicg.no

19. Thibodeau SN, French AJ, Roche PC, Cunningham JM, Tester DJ, Lindor NM, Möslein G, Baker SM, Liskay M, Burgart LJ, Honchel $\mathrm{R}$ and Halling KC. Altered expression of $\mathrm{hMSH} 2$ and $\mathrm{hMLH} 1$ in tumors with microsatellite instability and genetic alterations in mismatch repair genes. Cancer Res 1996; 56: 4836-4840.

20. de Leeuw WJF, Dierssen JW, Vasen HFA, Wijnen JT, Kenter GG, Meijers-Heijboer H, Brocker-Vriends A, Stormorken A, Møller P, Menko F, Cornelisse and Morreau H. Prediction of a mismatch repair gene defect by microsatellite instability and immunohistochemical analysis in endometrial tumors from HNPCC patients. J Pathol 2000; 192: 328-35.

21. Wijnen J, van der Klift H, Vasen, Meera Khan P, Menko F, Tops C, Meijers-Heijboer A, Lindhout D, Møller P and Fodde R. MSH2 genomic deletions are a frequent cause of HNPCC. Nat Genet 1998; 20: 326-328.

22. Eliason K, Hendrickson BC, Judkins T, Norton M, Leclair B, Lyon E, Ward B, Noll W and Scholl T. The potential for increased clinical sensitivity in genetic testing for polyposis colorectal cancer through the analysis of MYH mutations in North American patients. J Med Genet 2005; 42: 95-96.

23. Farrington SM, Tenesa A, Barnetson R, Wiltshire A, Prendergast J, Porteous M, Campbell $H$ and Dunlop MG. Germline susceptibility to colorectal cancer due to base-excision repair gene defects. Am J Hum Genet 2005; 77: 112-119.

24. Nielsen M, Franken PF, Reinards TH, Weiss MM, Wagner A, van der Klift H, Kloosterman S, Houwing-Duistermaat JJ, Aalfs CM, Ausems MG, Brocker-Vriends AH, Gomez Garcia EB, Hoogerbrugge N, Menko FH, Sijmons RH, Verhoef S, Kuipers EJ, Morreau H, Breuning MH, Tops CM, Wijnen JT, Vasen HF, Fodde R and Hes FJ. Multiplicity in polyp count and extracolonic manifestations in 40 Dutch patients with MYH associated polyposis coli (MAP). J Med Genet 2005; 42(9): e54. 